\title{
Factores asociados al resultado de tratamiento no exitoso de pacientes con tuberculosis sensible en Paraguay
}

\author{
Ivonne Montiel, ${ }^{1}$ Edith Alarcón, ${ }^{+}$Sarita Aguirre, ${ }^{1}$ Guillermo Sequera ${ }^{2}$ y Diana Marín ${ }^{3}$
}

Forma de citar

Montiel I, Alarcón E, Aguirre S, Sequera G, Martín D. Factores asociados al resultado de tratamiento no exitoso de pacientes con tuberculosis sensible en Paraguay. Rev Panam Salud Publica. 2020;44:e89. https://doi.org/10.26633/RPSP.2020.89

RESUMEN

Objetivo. Determinar los factores asociados con el tratamiento no exitoso en pacientes diagnosticados con tuberculosis (TB) sensible en Paraguay.

Métodos. Estudio de cohorte retrospectivo, con datos recolectados del programa nacional entre enero de 2016 y marzo de 2017. Se definió tratamiento no exitoso como pacientes que se perdieron en el seguimiento, fracasaron en el tratamiento o fallecieron. Los factores asociados con tratamiento no exitoso se determinaron con regresión de Poisson; se estimó el riesgo relativo (RR) y el intervalo de confianza de 95\% (IC95\%) ajustados por región sanitaria.

Resultados. Se estudiaron 3034 casos: 2189 (72,1\%) tuvieron tratamiento exitoso (curados: 1221 [40,2\%], tratamiento completo: 968 [31,9\%]) y $845(27,9 \%)$ presentaron tratamiento no exitoso (fracaso: 40 [1,3\%], pérdida de seguimiento: 466 [15,4\%] y fallecidos: 339 [11,2\%]). Los factores asociados a tratamiento no exitoso fueron hombres $1,28(1,14-1,42)$, indígenas $1,30(1,09-1,54)$, sin reporte de área de residencia 1,27 (1,02-1,57), coinfección TB/VIH 1,97 (1,63-2,38), adicción a drogas ilícitas 1,38 (1,16-1,63), consumo de alcohol 1,25 (1,02-1,52), ser previamente tratado 1,23 $(1,10-1,38)$ y sin datos de supervisión del tratamiento 4,92 (3,69- 6,56). Como factores protectores se consideran ser persona privada de libertad 0,65 (0,47- 0,89), así como la comorbilidad TB/diabetes $0,80(0,67-0,95)$.

Conclusión. Paraguay presenta un alto porcentaje de tratamiento no exitoso en casi todo el país, sin alcanzar la meta propuesta por la Organización Mundial de la Salud. Los factores de riesgo asociados como infección por el virus de la inmunodeficiencia humana $(\mathrm{VIH})$, consumo de drogas licitas e ilícitas y el ser indígena revela que es necesario revisar las estrategias dirigidas con abordaje interinstitucional.

Palabras clave Tuberculosis; factores de riesgo; VIH; grupos de población; investigación operativa; Paraguay.

En el 2017, se estimaron 10,4 millones de casos nuevos de tuberculosis (TB) con 1,7 millones de muertes en el mundo (1). Tres años antes, la Organización Mundial de la Salud (OMS) lanzó la Estrategia fin a la TB con metas a cumplir en el 2035; entre estas, reducir en $90 \%$ la tasa de incidencia de tuberculosis respecto del 2015. No obstante, el cumplimiento de esta meta se

\footnotetext{
1 Programa Nacional de Control de la Tuberculosis, Ministerio de Salud Pública y Bienestar Social, Asunción, Paraguay. $\bowtie$ Ivonne Montiel, ivonenfuna@gmail. com

2 Dirección de Vigilancia de la Salud, Ministerio de Salud Pública y Bienestar Social, Asunción, Paraguay.
}

ve comprometido por el alto porcentaje de tratamiento no exitoso que incrementa la probabilidad de desarrollar resistencia y predispone a mayor transmisión en la comunidad (2).

En 2016, la condición de egreso de tratamiento no exitoso fue de $18 \%$ en todo el mundo, con un porcentaje mayor en la Región de las Américas (24\%), debido principalmente a pérdidas en

\footnotetext{
3 Universidad Pontificia Bolivariana, Medellín, Colombia.

† Fallecida el 27 de agosto de 2019.
} 
el seguimiento y casos no evaluados. En Paraguay, en el 2015 se notificaron 2326 casos nuevos con TB sensible al Programa Nacional de Control de Tuberculosis (PNCT). Setenta por ciento de los pacientes tuvieron un tratamiento exitoso (3). Estas cifras se mantienen sin variaciones desde el año 2012 (3) y dista de la meta global de $90 \%$ (2).

El bajo porcentaje de tratamiento exitoso puede deberse también a la mortalidad de los pacientes con TB (4). Si bien las enfermedades respiratorias son la cuarta causa de mortalidad en el país, la TB ocupa el puesto 14 en las causas de muerte (4, 5). En Paraguay, en el año 2015 falleció $9,9 \%$ de los pacientes con tuberculosis. De estos, 23\% tenía coinfección por TB/VIH (4-6); para ese mismo año, la tasa de incidencia de VIH fue de 20,6 por cada 100000 habitantes (6). Se sabe que la posibilidad de un desenlace no exitoso en el tratamiento para TB aumenta de manera significativa en individuos con serología positiva para $\mathrm{VIH}$, migrantes, usuarios de drogas intravenosas, abusadores de drogas y personas en situación de calle $(7,8)$.

Si bien el país ha recibido apoyo del Fondo Mundial durante casi una década, aún no se alcanzan las metas establecidas por la OMS y se desconocen cuáles son los factores asociados con ello. El objetivo de esta investigación fue determinar los factores asociados con el tratamiento no exitoso en pacientes con diagnóstico de TB sensible en Paraguay.

\section{MATERIALES Y MÉTODOS}

\section{Diseño}

Investigación operativa que utilizó un diseño de cohorte retrospectivo para determinar los factores asociados con el tratamiento para tuberculosis sensible no exitoso. Se utilizaron los datos recolectados de manera sistemática en el programa nacional de Paraguay.

\section{Escenario del estudio}

Paraguay es un país de América del Sur con una población de 7052983 habitantes (9). El territorio se divide en dos regiones (oriental y occidental), con 14 y tres departamentos cada una, respectivamente. La capital del país es Asunción.

El Ministerio de Salud Pública y Bienestar Social (MSPBS) es el ente rector en materia de salud, ejerce los roles de rectoría, financiamiento y prestación de servicios. Esta última está dada por 18 regiones sanitarias constituidas por los departamentos y la capital del país.

El Programa Nacional de Control de la Tuberculosis (PNCT) depende de la Dirección General de Vigilancia de la Salud (DGVS) del MSPBS, y es la institución que dicta y supervisa el cumplimiento de las normativas para el manejo clínico y programático de la tuberculosis en el país. Además, realiza seguimiento, monitoreo y control de calidad de la información recolectada de manera estandarizada desde el nivel local hasta el nivel nacional.

\section{Población de estudio}

En el estudio se incluyeron todos los casos nuevos y previamente tratados, con diagnóstico confirmado de tuberculosis, que hayan iniciado o no tratamiento con fármacos de primera línea desde enero de 2016 hasta marzo de 2017 y que, además, contaban con condición de egreso del tratamiento. Se excluyeron los pacientes informados como "no evaluados" en el resultado del tratamiento para $\mathrm{TB}$, de acuerdo con las definiciones y marco de información de la OMS (10).

\section{Recolección de datos y variables}

Los datos se obtuvieron de la base del PNCT y se organizaron en Microsoft Excel ${ }^{\circledast}$ (Microsoft Corporation, Redmond, Washington, Estados Unidos). Las variables recolectadas incluyen las sociodemográficas y las clínicas.

Las variables sociodemográficas son la edad (años cumplidos), sexo (masculino, femenino), población indígena (sí, no), y área de residencia (rural, urbana).

Las variables clínicas son: forma clínica (pulmonar, extrapulmonar), paciente previamente tratado (sí, no), método diagnóstico (baciloscopia, cultivo, Xpert, clínica), tratamiento directamente observado (TDO) (sí, no), diabetes mellitus (sí, no), hábito de fumar (sí, no), haber tenido contacto con caso con TB (sí, no), persona privada de la libertad (PPL) (sí, no), coinfección TB/VIH (sí, no), adicción a drogas ilícitas (sí, no), y consumo de alcohol (sí, no).

En el estudio se utilizaron las definiciones de la OMS (10) que usa el PNCT. Para el análisis bivariado y multivariado, "tratamiento exitoso" fue curado y tratamiento completo, $\mathrm{y}$ "tratamiento no exitoso" fue fracaso del tratamiento, pérdida en el seguimiento y fallecido.

\section{Análisis de datos}

Los análisis se hicieron en SPSS ${ }^{\circledR} 22$ (IBM Corp. Released Armonk, NY: IBM Corp) y Stata ${ }^{\circledR}$ MP / 15 (StataCorp LP, College Station, Texas, Estados Unidos). Se presentaron frecuencias y porcentajes para las variables cualitativas y para las numéricas la mediana y el rango intercuartílico (RIC: Q1-Q3).

Se calculó el porcentaje de tratamiento no exitoso según la región sanitaria y el intervalo de confianza con el método de score de Wilson), estimado en OpenEpi. Se utilizó la prueba de chi cuadrado y la prueba exacta de Fisher en el análisis bivariado; se consideró como principal variable desenlace el tratamiento no exitoso, y aquellas variables que tuviesen un valor de $P$ bilateral menor a 0,25 se incluyeron en el análisis multivariado, donde se utilizó una regresión de Poisson. El riesgo relativo crudo (RRC) y el riesgo relativo ajustado (RRA) se estimaron con el respectivo intervalo de confianza de $95 \%$, ajustando los errores estándar por región sanitaria debido al efecto cluster. Se consideró significativo un valor de $P$ bilateral $<0,05$.

\section{Consideraciones éticas}

Se obtuvo autorización escrita del PNCT para utilizar la base de datos nacional desde enero de 2016 hasta marzo de 2017. Además, el protocolo fue aprobado por el Comité de Ética en Investigación del Laboratorio Central de Salud Pública del Paraguay y por el Comité de Revisión Ética de la Organización Panamericana de la Salud (PAHOERC).

Debido a la naturaleza retrospectiva y al empleo de datos ya registrados en la base de datos, no fue necesario el consentimiento informado de los pacientes. La base de datos fue 
FIGURA 1. Distribución porcentual de resultados de tratamiento no exitoso de pacientes de tuberculosis sensible según la región sanitaria, Paraguay, 2016

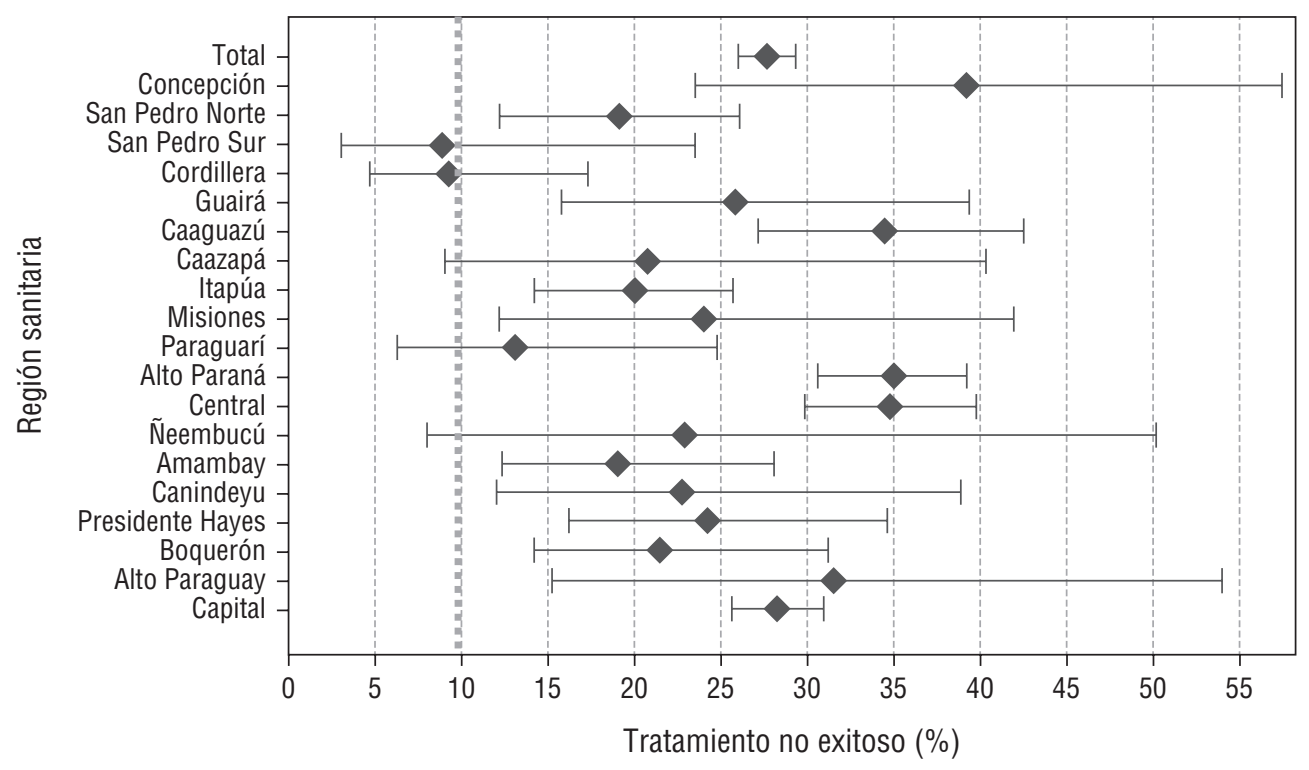

Fuente: elaboración propia a partir de los resultados presentados.

codificada y se omitió cualquier información que permitiera identificar a las personas.

\section{RESULTADOS}

En el período de estudio, se informó un total de 3390 casos de tuberculosis al PNCT. De estos, se excluyó el diagnóstico de TB en $48(1,4 \%), 25(0,7 \%)$ no tenían TB sensible, 240 (7\%) no presentaban condición de egreso y $43(1,2 \%)$ fueron casos no evaluados. Por lo tanto, se incluyeron 3034 casos con TB sensible en el estudio.

Tres regiones sanitarias (Asunción, Alto Paraná y Central) concentraron 1928 casos (64\%). En el nivel nacional, 2189 (72,1\%) de los casos con TB sensible tuvieron un tratamiento exitoso, $1221(40,2 \%)$ curados y 968 (31,9\%) con tratamiento completo. Se registraron $845(27,9 \%)$ con tratamiento no exitoso, 40 (1,3\%) con tratamiento fracasado, $466(15,4 \%)$ de pérdida en el seguimiento y 339 (11,2\%) fallecidos. Se observó una desigualdad del tratamiento no exitoso por región sanitaria: en Concepción el tratamiento fracasado fue $1(3,6 \%)$ mientras que la pérdida en el seguimiento fue en $10(35,7 \%)$ personas; otras regiones con altos porcentajes de pérdida en el seguimiento fueron Alto Paraná 117 (24,9\%); Caaguazú 33 (22,8\%); Central 73 (20,2\%) y Canindeyú con 7 (20\%). La mayor mortalidad se observó en Alto Paraguay 5 (26,3\%) y Ñeembucú 3 (23,1\%) (figura 1).

Los porcentajes más altos de tratamiento no exitoso se observaron en pacientes de sexo masculino, en mayores de 55 años, en indígenas, en pacientes con TB extrapulmonar, con coinfección con VIH, previamente tratados y con antecedentes de consumo de alcohol sin contacto previo con alguien con TB (cuadros 1 y 2).

Los pacientes que presentaron mayor riesgo de tratamiento no exitoso fueron los mayores de 15 años, sexo masculino (RR: 1,28; IC95\%: 1,14-1,42), indígenas (RR: 1,30; IC95\%: 1,09-1,54), sin reporte de área de residencia (RR: 1,27; IC95\%: 1,02-1,57), coinfección TB/VIH (RR: 1,97; IC95\%: 1,63-2,38), con adicción a drogas ilícitas (RR: 1,38; IC95\%: 1,16-1,63), consumo de alcohol (RR: 1,25; IC95\%: 1,02-1,52), haber recibido tratamiento previo para TB (RR: 1,23; IC95\%: 1,10-1,38) y sin datos de supervisión del tratamiento (RR: 4,92; IC95\%: 3,69-6,56). Por otra parte, estar privado de la libertad (RR: 0,65; IC95\%: 0,47-0,89) y tener diabetes (RR: 0,80; IC95\%: 0,67-0,95) fueron factores protectores para tratamiento no exitoso de TB sensible (cuadro 3).

\section{DISCUSIÓN}

Este es el primer estudio realizado en Paraguay sobre los factores que se asocian a un tratamiento no exitoso de pacientes con tuberculosis sensible. Nuestro estudio mostró un alto porcentaje de tratamiento no exitoso que varía considerablemente por región sanitaria y que factores sociodemográficos y clínicos reportados antes en la literatura aumentan el riesgo de tener un tratamiento no exitoso.

En Paraguay, el porcentaje de pacientes que alcanzaron un tratamiento exitoso fue de $72,1 \%$, muy por debajo de la meta de $90 \%$ propuesta por la OMS, aunque similar a lo observado en el mundo (83\%) y en la Región de las Américas (76\%) (1). Con este resultado, es probable que se comprometa la calidad de vida de las personas y las comunidades, con un riesgo mayor para las personas con otras comorbilidades que afectan su salud tal y como lo mencionan Harries et al. (11).

La alta proporción que las pérdidas en el seguimiento y la mortalidad aportan al porcentaje de tratamiento no exitoso ha sido informada previamente (1, 12-15). En Paraguay, el porcentaje de tratamiento no exitoso varió de manera considerable por región sanitaria. Algunas regiones se caracterizan por la diversidad poblacional y están compuestas principalmente por población indígena y privada de la libertad; otras tienen baja densidad poblacional, un número mayor de casos de TB (16) y un porcentaje alto de acceso a los servicios de salud (91\%) 
CUADRO 1. Condición de egreso programático de pacientes con tuberculosis sensible según las características sociodemográficas, Paraguay (2016)

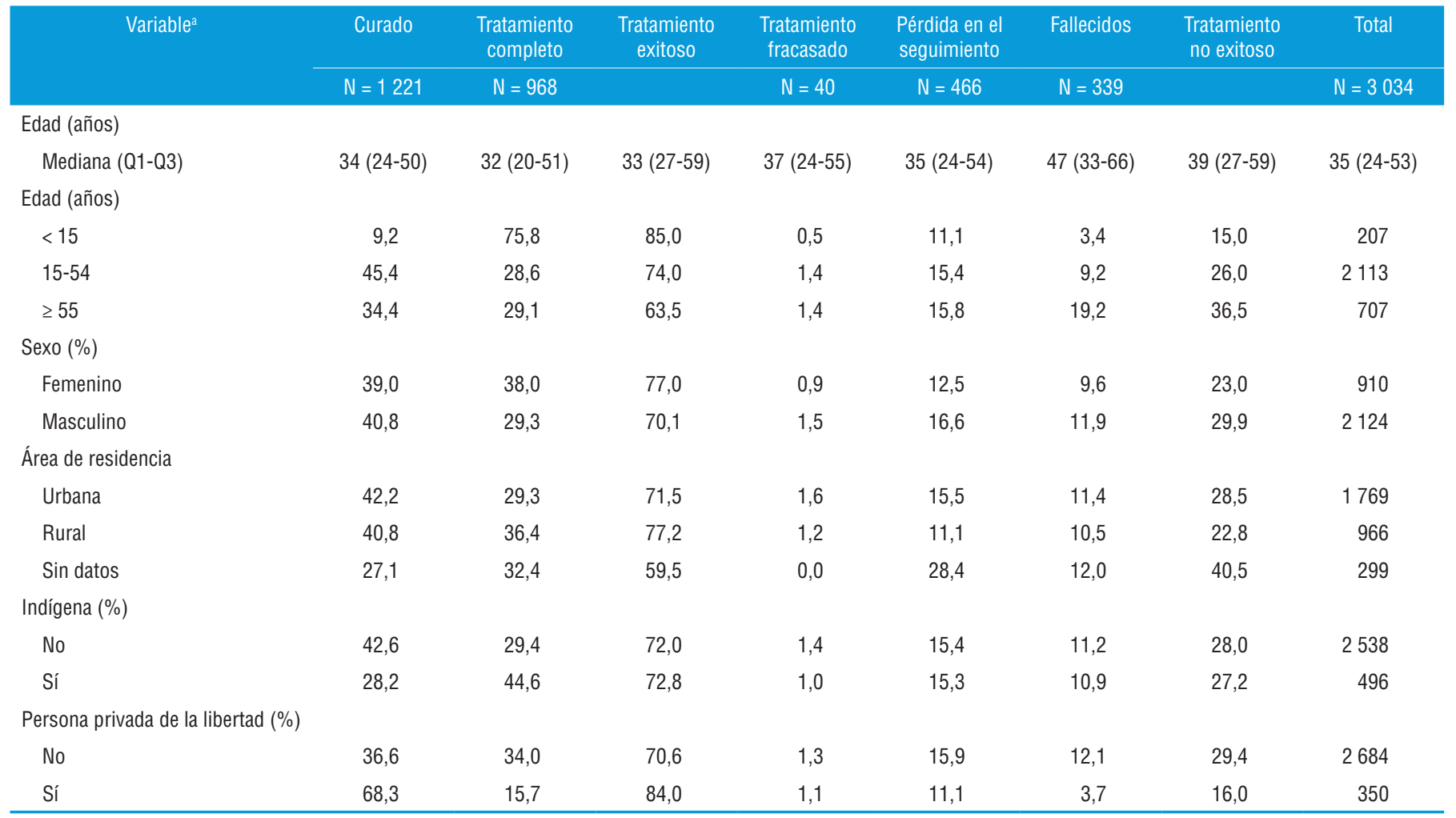

aTodas las variables arrojaron un valor de $P<0,01$ para la prueba de chi cuadrado de Pearson.

Q1, cuartil $1 ;$ Q3, cuartil 3

Fuente: elaboración propia a partir de los resultados presentados.

CUADRO 2. Condición de egreso programático de pacientes con tuberculosis sensible según las características clínicas, Paraguay (2016)

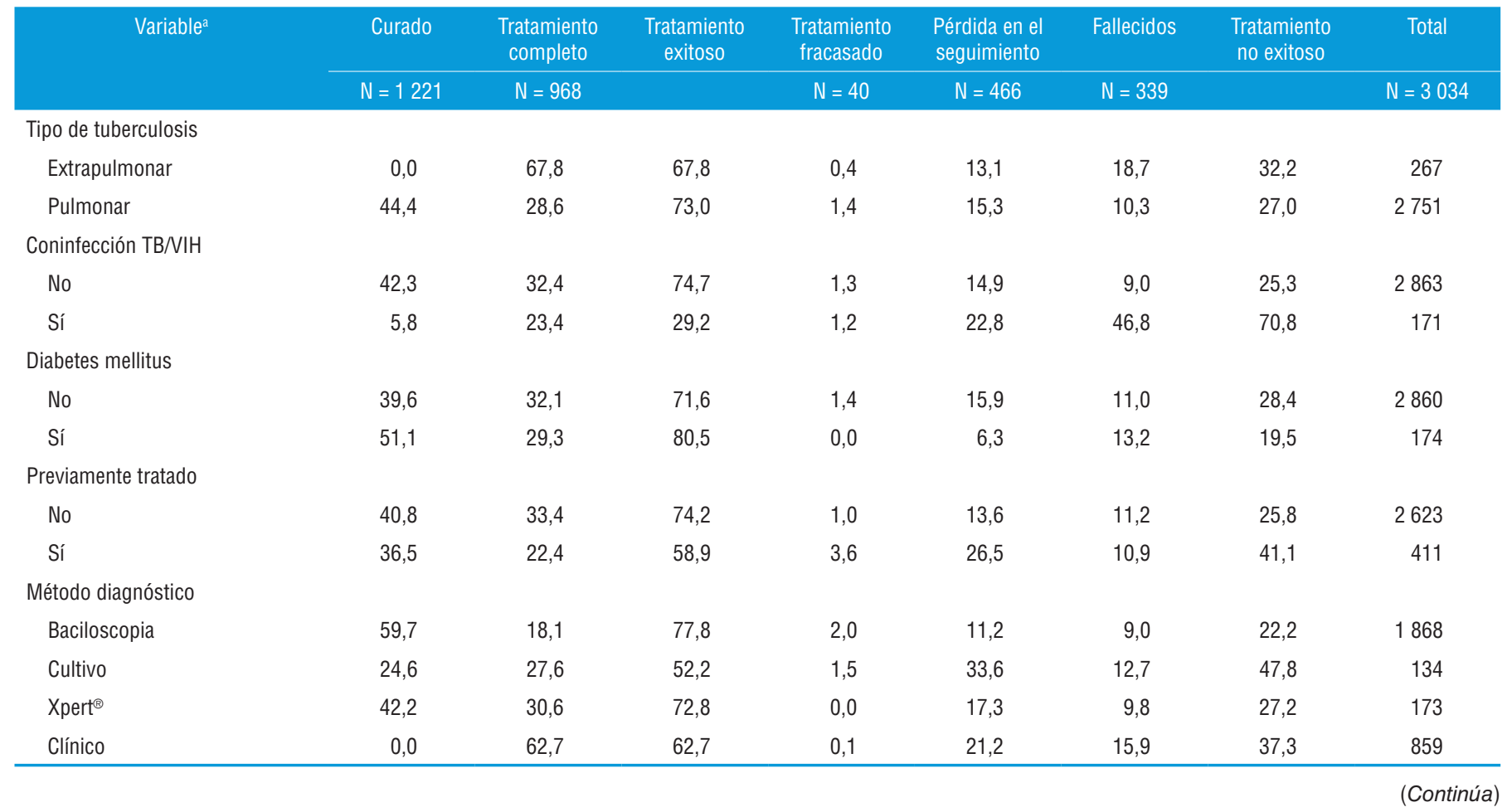


CUADRO 2. Condición de egreso programático de pacientes con tuberculosis sensible según las características clínicas, Paraguay (2016) (cont.)

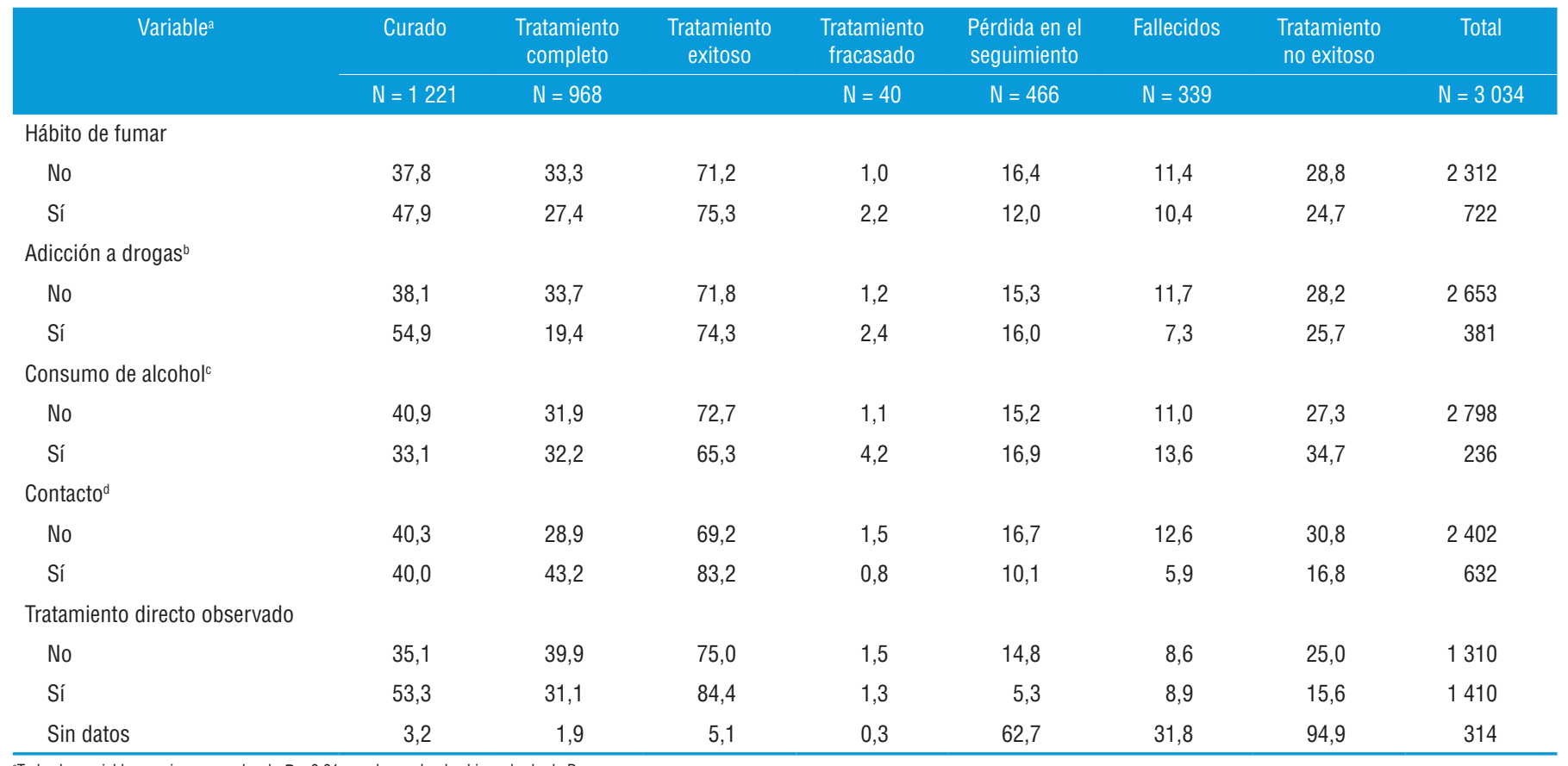

aTodas las variables arrojaron un valor de $P<0,01$ para la prueba de chi cuadrado de Pearson.

-Se considera como adicción a drogas el consumo de drogas ilícitas en el país como marihuana, crack y cocaína, entre otras, autorreportado por el caso al momento de la notificación.

Se considera consumo de alcohol el informado por la persona al momento de la notificación como caso de tuberculosis.

ES toda persona que haya estado expuesta a un caso índice bajo el mismo techo o dentre de un mismo espacio físico por 6 a 8 horas al día durante los últimos tres meses del diagnóstico del caso índice (34) TB/VIH, tuberculosis/virus de la inmunodeficiencia humana.

CUADRO 3. Factores sociodemográficos y clínicos asociados al resultado de tratamiento no exitosos de pacientes con tuberculosis sensible, Paraguay (2016)

\begin{tabular}{|c|c|c|c|c|c|c|c|c|}
\hline \multirow[t]{2}{*}{ Variables } & \multicolumn{2}{|c|}{ Tratamiento no exitoso } & \multicolumn{2}{|c|}{ Tratamiento exitoso } & \multicolumn{4}{|c|}{ Riesgo relativo } \\
\hline & $n$ & $\%$ & $n$ & $\%$ & Crudo & IC95\% & Ajustado & IC95\% \\
\hline \multicolumn{9}{|l|}{ Edad (años) } \\
\hline $15-54$ & 549 & 26,0 & 1564 & 74,0 & 1,73 & $(1,14-264)^{c}$ & 1,42 & $(1,13-1,79)^{d}$ \\
\hline$\geq 55$ & 258 & 36,5 & 449 & 63,5 & 2,44 & $(1,57-3,79)^{d}$ & 1,91 & $(1,35-2,70)^{d}$ \\
\hline \multicolumn{9}{|l|}{ Sexo } \\
\hline Masculino & 636 & 29,9 & 1488 & 70,1 & 1,3 & $(1,10-1,55)^{d}$ & 1,28 & $(1,14-1,42)^{d}$ \\
\hline \multicolumn{9}{|l|}{ Indígena } \\
\hline No & 710 & 28,0 & 1828 & 72,0 & 1 & & 1 & \\
\hline Sí & 135 & 27,2 & 361 & 72,8 & 0,97 & $(0,81-1,17)$ & 1,3 & $(1,09-1,54)^{d}$ \\
\hline \multicolumn{9}{|l|}{ Área } \\
\hline \multicolumn{9}{|c|}{ Persona privada de la libertad } \\
\hline No & 789 & 29,4 & 1895 & 70,6 & 1 & & 1 & \\
\hline Sí & 56 & 16,0 & 294 & 84,0 & 0,54 & $(0,42-0,70)^{d}$ & 0,65 & $(0,47-0,89)^{d}$ \\
\hline \multicolumn{9}{|l|}{ Tipo de tuberculosis } \\
\hline Extrapulmonar & 86 & 32,2 & 181 & 67,8 & 1 & & 1 & \\
\hline Pulmonar & 743 & 27,0 & 2008 & 73,0 & 0,84 & $(0,74-0,96)^{c}$ & 1,04 & $(0,95-1,13)$ \\
\hline
\end{tabular}


CUADRO 3. Factores sociodemográficos y clínicos asociados al resultado de tratamiento no exitosos de pacientes con tuberculosis sensible, Paraguay (2016) (cont.)

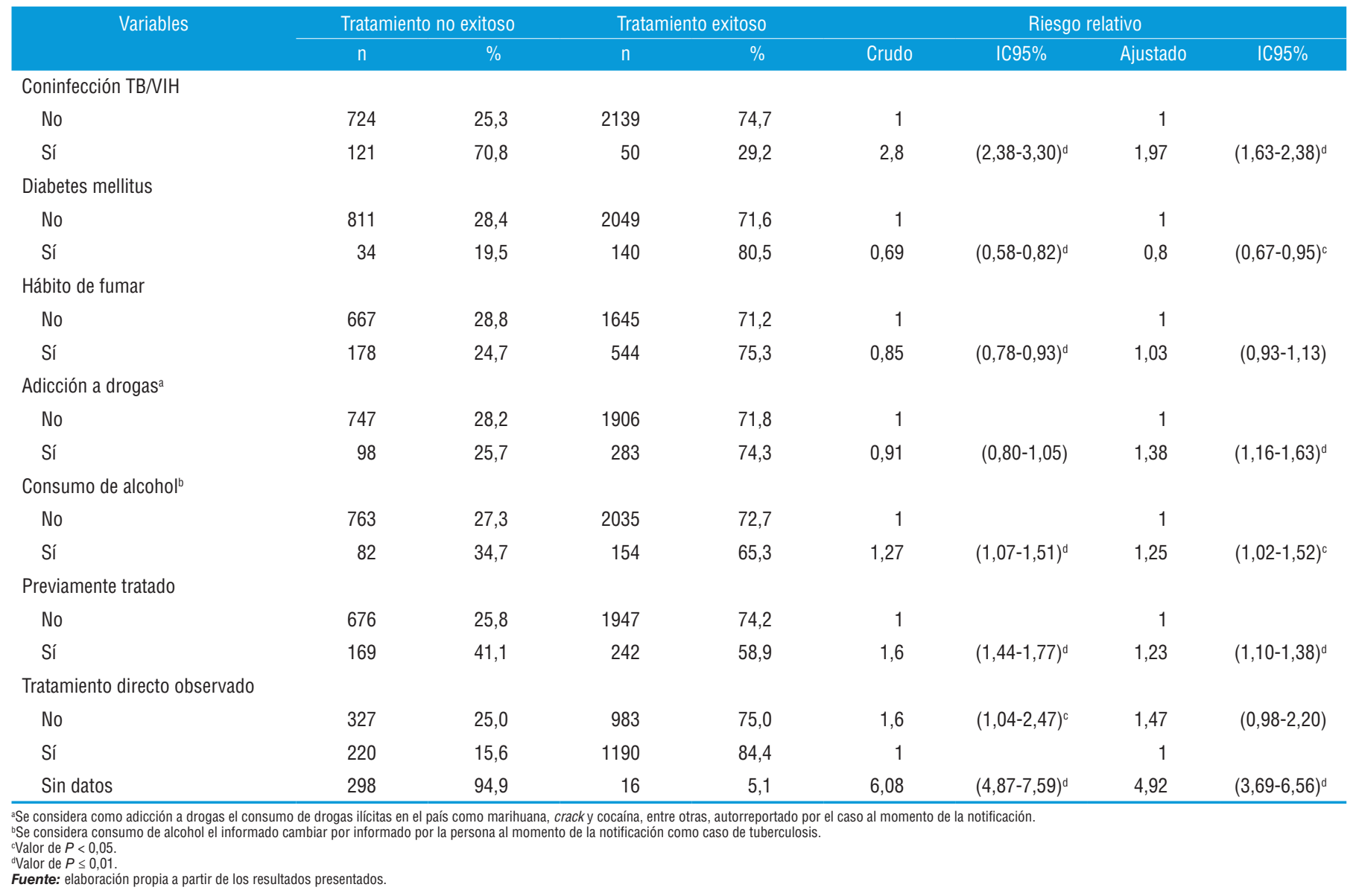

(17), lo cual contrasta con el $75 \%$ reportado en otros estudios (18). Es importante mencionar que, si bien la implementación de la estrategia de atención primaria busca acercar la atención sanitaria a la población, aún persisten dificultades por acceso geográfico e inadecuada infraestructura y equipamientos en los servicios de salud. Esto redunda en la pérdida de la continuidad en los procesos asistenciales (19), situación de la cual no está exenta la población afectada por TB (20).

En este estudio se ha evidenciado que el tratamiento no exitoso se asocia a factores como ser mayor a 55 años, similar a lo que otros estudios describen probablemente por comorbilidades asociadas en estas edades (21). También se encontró que el sexo masculino aumenta en $38 \%$ el riesgo de tratamiento no exitoso; esto podría deberse a una susceptibilidad biológica y a una menor notificación de tuberculosis en las mujeres por causas socioeconómicas y culturales $(21,22)$.

La población indígena resultó ser otro factor importante para un tratamiento no exitoso, dado que aumenta en $30 \%$ el riesgo de egresar sin éxito del tratamiento. En Paraguay, la población indígena representa 1,6\% de la población total y, en nuestro estudio, $40,5 \%$ de los indígenas egresaron con tratamiento no exitoso. Según una revisión sistemática reciente sobre los determinantes de la TB en pueblos indígenas, la carga de la enfermedad es desproporcionada, y los determinantes sociales como el consumo de alcohol, el hacinamiento, el abuso de sustancias y la presencia de comorbilidades como VIH y diabetes mellitus son más altos en poblaciones indígenas que no indígenas (23). Además, sus actividades económicas conllevan a un flujo migratorio alto (24) que, aunado a las barreras geográficas, lingüísticas y culturales, contribuyen al bajo control de la TB (23).

Como bien lo mencionan Cormier et al., existe poca evidencia que respalde enfoques específicos en la población indígena para incidir en los determinantes que contribuyen en la carga excesiva de la TB en la mencionada población. Solo confirman que hubo viabilidad y aceptabilidad de programas preventivos pero no abordan la eficacia de los mismos (23); es por ello que las estrategias que den continuidad al tratamiento en estas poblaciones es una tarea pendiente del país para el mayor control de la enfermedad, con implementación de estrategias con abordajes interculturales.

A diferencia de otros estudios donde no hubo asociación estadística significativa en relación al área de residencia (25), los pacientes que no cuentan con este reporte se han asociado a un tratamiento no exitoso en este estudio. Es probable que se trate de un déficit en el reporte, pues un porcentaje alto de los casos analizados no contaban con reporte de área de residencia.

Con el objetivo de fortalecer la Estrategia de tratamiento acortado y directamente observado (DOTS/TAES) y el proyecto de prevención y control de la TB en centros penitenciarios, Paraguay obtuvo dos financiamientos más del Fondo Mundial en el 
año 2009 (16). Con la implementación iniciada en el año 2010, se establecieron intervenciones diferenciadas de prevención y control con la instalación de laboratorios de diagnóstico gratuito, aislamiento de casos nuevos, mejoras en la alimentación, disminución del hacinamiento y prevención de las adicciones (26). Así, desde 2011 se observa la disminución progresiva de la incidencia (5), aunque la presentación de casos positivos nuevos $(79 \%)$ demuestra un alto grado de transmisibilidad de la enfermedad (5). En el presente estudio, las PPL tuvieron menor riesgo de tratamiento no exitoso, tal vez por la mejora de las condiciones de prestación sanitaria en las prisiones, así como la aplicación de la estrategia de tratamiento directamente observado.

La tuberculosis constituye una de las infecciones oportunistas que más afectan a las personas con VIH y se asocia con la mayor proporción de tratamiento no exitoso, probablemente debido a la diferencia en mortalidad en pacientes con VIH $(46,8 \%)$ en comparación con la población con serología negativa (9,0\%). En las Américas, 56,3\% de los pacientes con VIH positivo alcanzaron a egresar con éxito en el tratamiento (27), mientras que en Paraguay este porcentaje fue de $29,2 \%$. Si bien la tamización para VIH en pacientes con TB pasó de 33\% en el 2010 a 91\% en 2016, aún existe un alto porcentaje de diagnóstico tardío (32\%) (28). Esto se debe tal vez a que el país solo cuenta con 13 servicios de atención integral (SAI) para atención a las personas que viven con VIH, lo cual les obliga a trasladarse hasta el nivel nacional para recibir atención. Esta falta de articulación entre programas también disminuye el control de la TB.

En Paraguay, el abordaje de la comorbilidad TB y diabetes como estrategia se implementó por primera vez en 2014 (26). Como en otros países de la Región (27) se ha incluido en los formularios del sistema de registro la variable sobre diabetes para obtener datos aproximados de la prevalencia de diabetes en personas con TB. Si bien el campo es de obligatoria notificación, el porcentaje de datos perdidos y, posiblemente, el desconocimiento que tiene la persona de su condición, hace que haya un subreporte de la enfermedad, esto podría explicar el nivel de protección para tratamiento no exitoso. Otra posible explicación podría ser el mayor autocuidado de su salud en esta población. No obstante, no se cuenta con información del control de la glucemia como factor asociado con tratamiento exitoso (29). Por otra parte, la ausencia de datos sobre la prevalencia de diabetes en población general limita la comparación con otros estudios.

El hábito de fumar, el consumo de alcohol y el abuso de drogas se han asociado tanto con la incidencia de tuberculosis como con resultados negativos en su tratamiento, debido sobre todo a la falta de adherencia y consecuente abandono $(30,31)$. En nuestro estudio, no encontramos asociación con el hábito de fumar, lo cual podría deberse a un sesgo por la ausencia de información del tiempo de consumo y el número de cigarrillos que empezó a recolectarse de manera sistemática en el país a partir del 2019. Por otra parte, la asociación con el consumo de alcohol y el abuso de drogas podría deberse a características socioeconómicas y culturales con mayor prevalencia de consumo en comparación con la población general (23). Se requieren en el país estudios nacionales actualizados sobre este problema de salud pública, así como de estrategias programáticas que aborden estas adicciones para aumentar el alcance del tratamiento exitoso.

Como era de esperarse, en comparación con los nuevos, los pacientes previamente tratados tuvieron mayor riesgo de tratamiento no exitoso $(32,33)$. Esto podría deberse al desarrollo de resistencia por la exposición previa, así como deficiencias en el programa para la detección oportuna de la población con riesgo de falla en el tratamiento. Dichas deficiencias también se ven reflejadas en el manejo sistemático de la información; por ejemplo, la falta de información de modalidad de tratamiento directamente observado se asoció con un tratamiento no exitoso pese a que desde el 2013 se ha expandido a todos los servicios del país (16).

Este estudio tiene varias limitaciones. En primer lugar, se utilizó la base de datos del programa como principal fuente de información, y esta tenía datos incompletos. En segundo lugar, el estudio tuvo un diseño retrospectivo, por lo que no se midieron potenciales confusores; entre ellos el nivel educativo, la situación laboral, la condición psicológica y la oportunidad en el acceso al tratamiento. Por último, dado que algunos factores como la comorbilidad por DM y el uso de sustancias ilícitas y lícitas fueron autorreportados, podría haber sesgo de información.

\section{CONCLUSIÓN}

El tratamiento exitoso de la Tuberculosis sensible en el Paraguay se ha mostrado muy por debajo de la meta propuesta por la OMS de $90 \%$. El mayor riesgo de tratamiento no exitoso se presentó en individuos de sexo masculino, indígenas, sin reporte de área de residencia, coinfección TB/VIH, adicción a drogas ilícitas, alcoholismo, tratamiento previo y ausencia de reporte de supervisión del tratamiento. Los factores protectores fueron ser PPL y tener diabetes. Este estudio revela que es preciso revisar las estrategias dirigidas a poblaciones vulnerables con abordaje interinstitucional, multisectorial e intercultural con énfasis en la coinfección TB/VIH, el abuso de sustancias y otras comorbilidades.

Contribuciones de los autores. IM, EA, SA, GS y DM concibieron el estudio original, planificaron los experimentos, recolectaron y analizaron los datos, interpretaron los resultados, escribieron y revisaron el manuscrito. Todos los autores han leído y aprobado el manuscrito y han contribuido significativamente al trabajo.

Agradecimientos. Esta investigación se llevó a cabo mediante la Iniciativa de Capacitación Estructurada en Investigación Operativa (SORT IT, por sus siglas en inglés), una alianza mundial dirigida por el Programa Especial de Investigación y Capacitación de Enfermedades Tropicales de la Organización Mundial de la Salud (OMS/TDR) y el Departamento de Enfermedades Transmisibles y Determinantes Ambientales de la Salud de la Organización Panamericana de la Salud (OPS).

Financiamiento. Se obtuvo financiamiento de la oficina subregional Andina de la OPS. Los financiadores no desempeñaron ningún papel en el diseño del estudio, la recopilación y análisis de los datos, la decisión de publicar ni la elaboración del artículo.

Conflicto de intereses. Ninguno declarado por los autores.

Declaración. Las opiniones expresadas en este manuscrito son únicamente responsabilidad de los autores y no reflejan necesariamente los de la Revista Panamericana de Salud Pública o la Organización Panamericana de la Salud. 


\section{REFERENCIAS}

1. Organización Mundial de la Salud. Informe mundial sobre la tuberculosis. Gineabra: OMS; 2017. Disponible en: https://www.who. $\mathrm{int} / \mathrm{tb}$ /publications/global_report/gtbr2017_main_text.pdf

2. Organización Mundial de la Salud. Estrategia de Fin a TB: objetivos e indicadores. Ginebra: OMS; 2015. Disponible en: https://www. who.int/tb/strategy/end-tb/es/

3. World Health Organization. Tuberculosis data. Geneva: WHO; 2020. Disponible en: http://www.who.int/tb/data/en/

4. Medina A, López L, Martínez C, Aguirre S, Alarcón E. Factores asociados a la mortalidad por tuberculosis en Paraguay, 2015-2016. Rev Panam Salud Publica 2019;43:e102. Disponible en: https://www. ncbi.nlm.nih.gov/pmc/articles/PMC6922078/

5. Ministerio de Salud Pública y Bienestar Social de Paraguay, Organización Panamericana. Programa Nacional de Control de Tuberculosis Paraguay. Nota conceptual de tuberculosis para el Fondo Mundial, 2019-2021. Asunción: MSPBS y OPS; 2018.

6. Ministerio de Salud Pública y Bienestar Social de Paraguay. Informe sobre la situación del VIH en Paraguay, 2017. Disponible en: https://www.mspbs.gov.py/pronasida/informes-varios.html

7. Nunes C, Duarte R, Veiga AM, Taylor B. Who are the patients that default tuberculosis treatment? Space matters! Epidemiol Infect. 2017;145(6):1130-4.

8. Gómez LM, Paniagua-Saldarriaga LA, Richert Q, Keynan Y, Montes F, López L et al. Homelessness and HIV: a combination predictive of poor tuberculosis treatment outcomes and in need of innovative strategies to improve treatment completion. Am J Trop Med Hyg. 2019;100(4):932-9.

9. Dirección General de Estadística, Encuestas y Censos de Paraguay (DGEEC). Proyección de la población nacional, áreas urbana y rural por sexo y edad, 2000-2025. Asunción: DGEEC; 2015. Disponible en: https://www.dgeec.gov.py/Publicaciones/Biblioteca/ proyeccion $\% 20$ nacional/Estimacion $\% 20 y \% 20$ proyeccion $\% 20$ Nacional.pdf

10. Organización Mundial de la Salud. Definiciones y marco de trabajo para la notificación de Tuberculosis - Revisión 2013. Ginebra: OMS; 2013. Disponible en: https://apps.who.int/iris/bitstream/ handle $/ 10665 / 111016 / 9789243505343$ spa.pdf;jsessionid=84AAD52B9A7ABC51EDC24B958A8C2823? sequence $=1$

11. Harries AD, Dlodlo RA, Brigden G, Mortimer K, Jensen P, Fujiwara PI, et al. Should we consider a «fourth 90» for tuberculosis? Int J Tuberc Lung Dis. 2019;23(12):1253-56. doi: 10.5588/ijtld.19.0471.

12. Amante TD, Ahemed TA. Risk factors for unsuccessful tuberculosis treatment outcome (failure, default and death) in public health institutions, Eastern Ethiopia. Pan Afr Med J. 2015;20:247. Disponible en: https://www.ncbi.nlm.nih.gov/pmc/articles/PMC448 4196/

13. Gebrezgabiher G, Romha G, Ejeta E, Asebe G, Zemene E, Ameni G. Treatment outcome of tuberculosis patients under directly observed treatment short course and factors affecting outcome in Southern Ethiopia: a five-year retrospective study. PloS One. 2016;11(2):e0150560.

14. Rodríguez E, Villarrubia S, Díaz O, Hernández G, Tello O. Situación de la tuberculosis en España. Casos de tuberculosis declarados a la Red Nacional de Vigilancia Epidemiológica en 2010. Bol Epidemiológico. 2012;20(3):26-32.

15. Rojas CM, Villegas SL, Piñeros HM, Chamorro EM, Durán CE, Hernández EL, et al. Clinical, epidemiological and microbiological characteristics of a cohort of pulmonary tuberculosis patients in Cali, Colombia. Biomédica. 2010;30(4):478-81.

16. Ministerio de Salud Pública y Bienestar Social de Paraguay. Programa Nacional de Control de Tuberculosis. Plan estratégico de la respuesta nacional a la tuberculosis en Paraguay, 2016-2020 Disponible en: http://vigisalud.gov.py/webdgvs/views/paginas/webtb/ docs/plan_estrategico_nacional_tb.pdf

17. Dirección General de Estadística, Encuestas y Censos de Paraguay (DGEEC). Encuesta permanente de hogares continua 2020. Disponible en: https://www.dgeec.gov.py/microdatos/EncuestaPermanente-de-Hogares-Continua.php

18. Dirección General de Estadística, Encuestas y Censos de Paraguay (DGEEC). Atlas de las Comunidades Indígenas en el Paraguay.
Disponible en: https://www.dgeec.gov.py/Publicaciones/Biblioteca/Web\%20Atlas\%20Indigena/Atlasindigena.htm

19. Dullak R, Rodriguez-Riveros MI, Bursztyn I, Cabral-Bejarano MS, Ruoti M, Paredes ME, et al. Atención primaria en salud en Paraguay: panorámica y perspectiva. Cienc Saude Coletiva. 2011;16(6):2865-75. Disponible en: http:/ / www.scielo.br/scielo.php?script=sci_arttext\&pid=S1413-81232011000600024\&lng=es\&tlng=es

20. Ministerio de Salud Pública y Bienestar Social de Paraguay. Plan Local Salud de Concepción 2014-2016. Asunción: MSPBS; 2014. Disponible en: https://www.cird.org.py/institucional/documentos/ Plan_Local_Salud_Concepcion.pdf

21. Wen Y, Zhang Z, Li X, Xia D, Ma J, Dong Y, et al. Treatment outcomes and factors affecting unsuccessful outcome among new pulmonary smear positive and negative tuberculosis patients in Anqing, China: a retrospective study. BMC Infect Dis. 2018;18:104. Disponible en: https://www.ncbi.nlm.nih.gov/pmc/articles/PM C5836329/

22. Neyrolles O, Quintana-Murci L. Sexual inequality in tuberculosis. PLoS Med [Internet]. 2009;6(12). Disponible en: https:/ /www.ncbi. nlm.nih.gov/pmc/articles/PMC2788129/

23. Cormier M, Schwartzman K, N'Diaye DS, Boone CE, dos Santos AM, Gaspar J, et al. Proximate determinants of tuberculosis in Indigenous peoples worldwide: a systematic review. Lancet Glob Health. 2019;7(1):e68-80. Disponible en: http://www.sciencedirect. com/science/article/pii/S2214109X18304352

24. Secretaría Técnica de Planificación del Desarrollo Económico y Social de Paraguay. Situación económica, social y ambiental en Paraguay, 2012. Disponible en: http://www.geam.org.py/v3/ cgp/files/2009/01/DIAGN\%25C3\%2593STICO-PAIS-UT-2012. pdf

25. Ukwaja KN, Alobu I, Gidado M, Onazi O, Oshi DC. Economic support intervention improves tuberculosis treatment outcomes in rural Nigeria. Int J Tuberc Lung Dis. 2017;21(5):564-570. doi: 10.5588/ijtld.16.0741

26. Ministerio de Salud Pública y Bienestar Social de Paraguay. Resumen de Gestión del Programa Nacional de Control de la Tuberculosis 2013-2017. Documento técnico. Asunción: MSPBS; 2017.

27. Organización Panamericana de la Salud. Tuberculosis en las Américas 2018. Washington D.C.: OPS; 2018. Disponible en: https:/ iris.paho.org/bitstream/handle/10665.2/49510/OPSCDE18036_ spa? sequence $=2$ \&isAllowed $=y$

28. Organización Panamericana de la Salud/Organización Mundial de la Salud. Plan Nacional de control de la coinfección tuberculosis/VIH (2017-2020). Disponible en: https:/ /www.paho.org/par/ index.php?option=com_docman\&view=document\&slug=plannacional-de-control-de-la-coinfeccion-tuberculosis-vih-2017-2020 \&layout=default\&alias=577-plan-nacional-de-control-de-la-coinfeccion-tuberculosis-vih-2017-2020\&category_slug=publicacionescon-contrapartes\&Itemid $=253$

29. Nascimento CV, Soares SM. Manejo integrado de tuberculose e diabetes: uma revisão integrativa. Rev Panam Salud Publica. 2018;43:1. Disponible en: http://iris.paho.org/xmlui/handle/123 $456789 / 49772$

30. Silva DR, Muñoz-Torrico M, Duarte R, Galvão T, Bonini EH, Arbex $\mathrm{FF}$, et al. Risk factors for tuberculosis: diabetes, smoking, alcohol use, and the use of other drugs. J Bras Pneumol. 2018;44(2):14552. Disponible en: https://www.ncbi.nlm.nih.gov/pmc/articles/ PMC6044656/

31. Przybylski G, Da browska A, Trzcin’ska H. Alcoholism and other socio-demographic risk factors for adverse TB-drug reactions and unsuccessful tuberculosis treatment - data from ten years' observation at the Regional Centre of Pulmonology, Bydgoszcz, Poland Med Sci Monit 2014;20:444-53. Disponible en: https://www.ncbi. nlm.nih.gov/pmc/articles/PMC3965286/

32. Rodríguez-Valín E, Villarrubia Enseñat S, Díaz García O, Martínez Sánchez EV. Risk factors for potentially unsuccessful results and mortality during tuberculosis treatment in Spain. Rev Esp Salud Publica. 201589(5):459-70. Disponible en: http://scielo. isciii.es / scielo.php?script=sci_abstract\&pid=S1135-57272015000 500004 
33. Ejeta E, Beyene G, Balay G, Bonsa Z, Abebe G. Factors associated with unsuccessful treatment outcome in tuberculosis patients among refugees and their surrounding communities in Gambella Regional State, Ethiopia. PLoS ONE. 2018;13(10). Disponible en: https://www.ncbi.nlm.nih.gov/pmc/articles/PMC6193657/

34. Ministerio de Salud Pública y Bienestar Social de Paraguay. Guía nacional para el manejo de la tuberculosis. Servicios de salud locales, distritales, regionales. Paraguay, 2018. Disponible en: https:// www.paho.org/par/index.php?option $=$ com_docman\&view $=$ do cument\&layout=default\&alias=576-guia-nacional-para-el-mane jo-de-la-tuberculosis-2017\&category_slug=publicaciones-con-contrapartes\&Itemid $=253$

Manuscrito recibido el 27 de julio de 2019. Aceptado para su publicación, tras revisión, el 10 de marzo de 2020.

\section{Factors associated with unsuccessful treatment of patients with drug-sensitive tuberculosis in Paraguay}

ABSTRACT Objective. To determine the factors associated with the unsuccessful treatment of patients diagnosed with drug-sensitive tuberculosis (TB) in Paraguay.

Methods. Retrospective cohort study, with data collected from the national program between January 2016 and March 2017. Unsuccessful treatment was defined as patients who were lost to follow-up, failed treatment, or died. Factors associated with unsuccessful treatment were determined using Poisson regression; the adjusted relative risk (RR) and 95\% confidence interval $(95 \% \mathrm{Cl})$ were estimated by health region.

Results. A total of 3034 cases were studied: 2189 (72.1\%) were successfully treated (cured: 1221 [40.2\%], complete treatment: 968 [31.9\%]) and 845 (27.9\%) were unsuccessfully treated (treatment failure: 40 [1.3\%], loss to follow-up: 466 [15.4\%] and deaths: 339 [11.2\%]). Factors associated with unsuccessful treatment were masculine sex 1.28 (1.14-1.42), indigenous descent 1.3 (1.09-1.54), lack of report of area of residence 1.27 (1.02- 1.57), TB/HIV coinfection 1.97 (1, 63- 2.38), illicit drug addiction 1.38 (1.16- 1.63), alcohol consumption 1.25 (1.02- 1.52), previous treatment 1.23 (1.10- 1.38) and lack of treatment monitoring data 4.92 (3.69- 6.56). Being deprived of liberty 0.65 (0.47- 0.89) and TB/diabetes comorbidity 0.80 (0.67- 0.95) were considered protective factors.

Conclusion. Paraguay has a high percentage of unsuccessful treatment in almost the entire country, without reaching the target proposed by the World Health Organization. Associated risk factors such as HIV, consumption of legal and illicit drugs, and being indigenous highlight the need to revise the treatment strategies with an inter-institutional approach.

Keywords $\quad$ Tuberculosis; risk factors; HIV; population groups; operations research; Paraguay. 